Quasi-monoenergetic proton acceleration from cryogenic hydrogen microjet by ultrashort ultraintense laser pulses

A. Sharma, Z. Tibai, J. Hebling, and J. A. Fülöp

Citation: Physics of Plasmas 25, 033111 (2018); doi: 10.1063/1.5003353

View online: https://doi.org/10.1063/1.5003353

View Table of Contents: http://aip.scitation.org/toc/php/25/3

Published by the American Institute of Physics

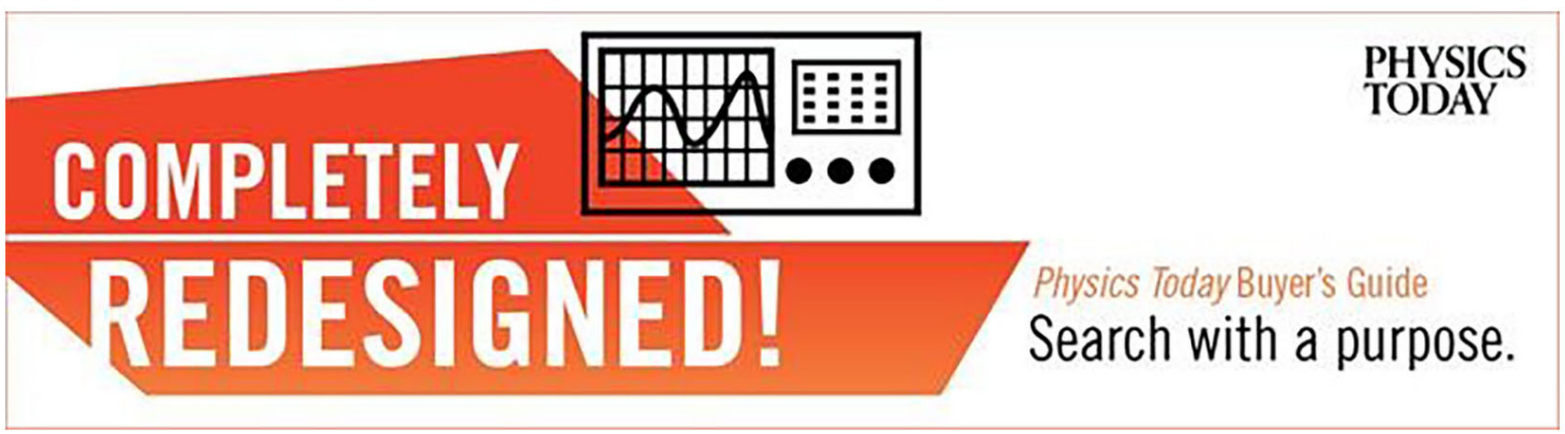




\title{
Quasi-monoenergetic proton acceleration from cryogenic hydrogen microjet by ultrashort ultraintense laser pulses
}

\author{
A. Sharma, ${ }^{1, a)}$ Z. Tibai, ${ }^{2}$ J. Hebling, ${ }^{2,3,4}$ and J. A. Fülöp ${ }^{1,3,4}$ \\ ${ }^{1}$ ELI-ALPS, ELI-HU Non-Profit Ltd., Dugonics ter 13, H-6720 Szeged, Hungary \\ ${ }^{2}$ Institute of Physics, University of Pécs, Pécs 7624, Hungary \\ ${ }^{3}$ Szentágothai Research Centre, University of Pécs, Pécs 7624, Hungary \\ ${ }^{4}$ MTA-PTE High-Field Terahertz Research Group, Pécs 7624, Hungary
}

(Received 5 September 2017; accepted 4 March 2018; published online 19 March 2018)

Laser-driven proton acceleration from a micron-sized cryogenic hydrogen microjet target is investigated using multi-dimensional particle-in-cell simulations. With few-cycle (20-fs) ultraintense (2-PW) laser pulses, high-energy quasi-monoenergetic proton acceleration is predicted in a new regime. A collisionless shock-wave acceleration mechanism influenced by Weibel instability results in a maximum proton energy as high as $160 \mathrm{MeV}$ and a quasi-monoenergetic peak at $80 \mathrm{MeV}$ for $10^{22} \mathrm{~W} / \mathrm{cm}^{2}$ laser intensity with controlled prepulses. A self-generated strong quasistatic magnetic field is also observed in the plasma, which modifies the spatial distribution of the proton beam. Published by AIP Publishing. https://doi.org/10.1063/1.5003353

\section{INTRODUCTION}

Monoenergetic ion beams play an essential role in many important applications such as fast ignition fusion and cancer therapy. ${ }^{1}$ Laser-driven ion acceleration attracts increasing attention nowadays since the acceleration gradient is at least four orders of magnitude higher than that of conventional methods. A number of novel mechanisms have been proposed for accelerating protons or heavier ions to high energies using the recently available high-intensity short-pulse lasers. The relativistically intense lasers can accelerate ion beams from plasmas to high energies $(>\mathrm{MeV})$ in extremely short distances. Recent development has demonstrated the laser-driven acceleration of protons via different mechanisms, e.g., target normal sheath acceleration (TNSA), ${ }^{2-9}$ radiation pressure acceleration (RPA), ${ }^{10-14}$ shock wave acceleration (SWA), ${ }^{15-18}$ and acceleration in a relativistic transparency regime, ${ }^{19-22}$ which are the centre of experiments and theoretical investigations.

Acceleration of protons to high energy by the laserplasma interaction is possible due to the production of hot electrons and the generation of very strong ambipolar fields. Hot electron sheath production requires a large value of $I \times \lambda_{L}^{2}$ and steep plasma density gradients. The ion energy scales approximately with the irradiance $\simeq\left(I \times \lambda_{L}^{2}\right)^{1 / 2}$, where $I$ and $\lambda_{L}$ are the laser intensity and wavelength, respectively. ${ }^{7}$ Despite significant progress, the requirements for applications in terms of ion energy, conversion efficiency, spectral width, brilliance, and suitability for high-repetition rate operations have not been achieved yet. To overcome the limitations of TNSA, recent studies using particle-in-cell (PIC) simulations have shown that using higher laser intensities and tailored high-density targets can access more favourable regimes of laser-driven ion acceleration. ${ }^{16,21,23}$

The maximum energies obtained under various experimental conditions are well understood. Low energy protons

\footnotetext{
${ }^{\text {a)} E m a i l: ~ a s h u t o s h . s h a r m a @ e l i-a l p s . h u ~}$
}

are produced in the outer regions around the focused laser beam with a lower electric field and a typical transversal diameter of several $100 \mu \mathrm{m}$. This enormous lateral extension is attributed to recirculating electrons which spread around the target, thus causing a decay of the acceleration field. Confining recirculating hot electrons by using mass-limited targets (MLTs), i.e., targets with transverse dimensions comparable to the laser focus $(10-20 \mu \mathrm{m})$, can enhance the electric fields and result in increased ion energies.

MLTs, such as cryogenic hydrogen microjets (CHMs), are highly appropriate to study alternative acceleration mechanisms. Cryogenic targets can also be better alternatives to solid foils because of their operation at high repetition rates. In a recent experiment with a CHM target using 700-fs frequency-doubled pulses from the Titan laser system at the Jupiter Laser Facility (Lawrence Livermore National Laboratory), the generation of a pure proton beam was demonstrated with a maximum energy of $2 \mathrm{MeV}^{24}$ Protons exhibiting a semi-Maxwellian spectrum were present in a higher number in the target surface normal direction, which is typical for TNSA. In the laser forward direction, a significantly modulated proton spectrum was observed in the highenergy part, showing a strong quasi-monoenergetic peak at $1.1 \mathrm{MeV}$, with an energy spread of $4 \%$ full width at half maximum (FWHM). This clearly indicated a non-TNSA acceleration mechanism. The laser-to-proton energy conversion was predicted to be most efficient when the target gets closer to the relativistic critical density $\gamma n_{c}{ }^{14}$ Here, $\gamma=\sqrt{\left(1+a_{L}^{2}\right)}$ is the relativistic factor of the laser with the normalised electric field $a_{L}=e E_{L} /\left(m_{e} \omega_{L} c\right)$, where $E_{L}$ is the electric field. $n_{c}$ is the critical density corresponding to the laser frequency $\omega_{L}, c$ is the speed of light, and $e$ and $m_{e}$ are the electronic charge and mass, respectively. Another recent investigation ${ }^{26}$ reported the experimental and simulation evidence for multi-MeV proton acceleration where the solid density CHM target has been deployed and the role of preplasma was also investigated. Recently, Gauthier et al. ${ }^{27}$ 
reported the high repetition rate $(1 \mathrm{~Hz}) 6.5 \mathrm{MeV}$ proton source from a CHM target, irradiated by $100 \mathrm{TW}$ laser pulses delivering $10^{13}$ protons $/ \mathrm{MeV} / \mathrm{sr} / \mathrm{min}$. Obst et al. ${ }^{28}$ reported efficient proton acceleration from the CHM target of cylindrical and planar shapes in an experiment with the $150 \mathrm{TW}$ Draco laser. In this experiment, protons up to $20 \mathrm{MeV}$ energy were produced with $10^{9}$ protons $/ \mathrm{MeV} / \mathrm{sr}$. This result illustrated for the first time that proton acceleration from CHM targets are of comparable performance to solid foil targets of micrometer range.

In this article, we investigate the proton acceleration by the interaction of high-intensity short laser pulses with a near-solid-density CHM of cylindrical shape, employing fully relativistic multi-dimensional particle-in-cell (PIC) simulations. The theoretical framework of previous studies ${ }^{26}$ is applied for ultra-relativistic laser intensities. Simulation results are in close agreement with the experiment and simulation evidence of Ref. 26. The validity of the model is justified by reproducing the experimental results of Gauthier et $a l .{ }^{24} \mathrm{~A}$ new regime of the interaction is proposed which utilises few-cycle (20 fs) high power (2 PW) laser pulses for high-energy proton acceleration with a quasi-monoenergetic feature. Such pulses will be available at ELI-ALPS ${ }^{25}$ in the near future. The proton acceleration mechanism is dominated by collisionless shock wave acceleration influenced by Weibel instability. The dependence of proton energy distribution on the preplasma scale length is also investigated and illustrated through numerical simulation.

\section{THEORETICAL MODEL}

In order to investigate the proton acceleration mechanism in CHM, we numerically model the interaction of an ultrashort-ultraintense laser field with near-solid-density plasma with the aid of multidimensional PIC simulations using the PIConGPU code. ${ }^{29}$ Figure 1(a) shows the scheme of the simulation model, which is similar to that used in the experiment of Gauthier et al. ${ }^{24}$ An intense laser pulse, propagating along the $y$-axis, is focused onto the CHM target with normal incidence. The laser is linearly polarized along the $\mathrm{x}$-direction. A laser pulse of $800 \mathrm{~nm}$ wavelength is considered which is Gaussian in space and time. The focused beam diameter at the target is $2 r_{0}=5.0 \mu \mathrm{m}$, the laser pulse duration

\section{CHM target}

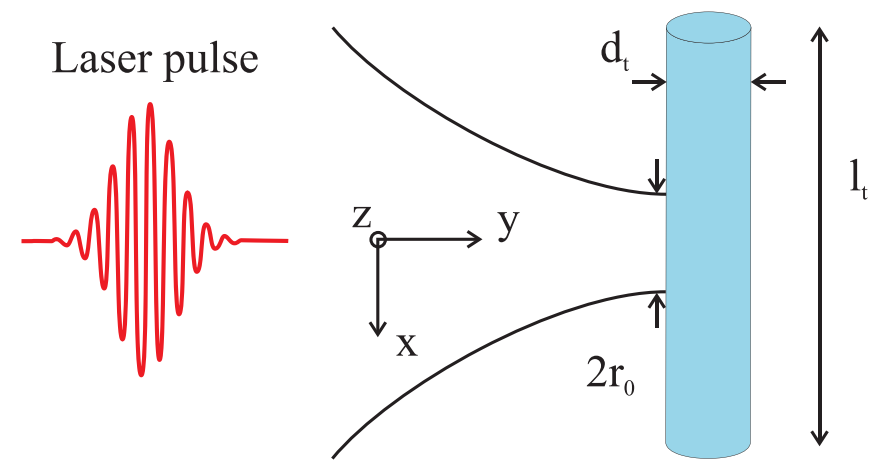

FIG. 1. Scheme of the setup for the interaction of a laser pulse with a preionised CHM target of cylindrical shape. is $20 \mathrm{fs}$, the peak laser intensity is about $10^{22} \mathrm{~W} / \mathrm{cm}^{2}$, and correspondingly, the normalised laser field is $a_{L}=68$. The proposed CHM plasma target is of cylindrical shape with a diameter of $d_{t}=2.5 \mu \mathrm{m}$. It was assumed to be nonmagnetised preionized with an initial density of the fully ionised plasma to be $6.96 \times 10^{22} \mathrm{~cm}^{-3}\left(\simeq 40 n_{c}\right)$, where $n_{c}$ is the critical density for $800 \mathrm{~nm}$ light. The computational domain is $10 \times 10 \times 10 \mu \mathrm{m}^{3}$ with a cell size of $10 \mathrm{~nm}$ (in longitudinal and transverse directions), and the time step is 16.7 As. Two particles per cell are considered for each species (electrons and protons). Periodic boundary conditions were used in the simulations in the transverse directions (along $x$ - and $z$-axes). The open/absorbing boundary conditions were used along the laser propagation direction ( $y$-axis) to reduce the computational time. The peak density in simulation increases up to 2-3 times the initial plasma density so the grid size and time step are chosen carefully to resolve the electron dynamics within the relativistic collisionless skin depth $\left(\simeq \gamma^{1 / 2} c / \omega_{p e}\right)$, where $\omega_{p e}$ is the electron plasma frequency.

When an intense laser field $\left(a_{L} \gg 1\right)$ interacts with the preionised plasma, the electrons acquire an exponential energy distribution with a mean energy of $k_{B} T_{e}$ $=m_{e} c^{2}\left(\sqrt{\left(1+a_{L}^{2}\right)}-1\right)$ due to ponderomotive acceleration. ${ }^{10}$ Here, $k_{B}$ is the Boltzmann constant and $T_{e}$ is the bulk electron temperature. With intensities of $I=10^{18}-10^{21} \mathrm{~W} / \mathrm{cm}^{2}$ available today, the ions hardly move in the electric field of the laser. As soon as the laser starts to tear off the electrons, its $e(\mathbf{v} \times \mathbf{B})$ force pushes electrons forward and the longitudinal electric field evolves due to the charge separation. This stationary (quasi-static) electric field can be as high as the electric field of the laser itself. The laser ponderomotive force $f_{L}$ responsible for the laser-matter interaction can be expressed as ${ }^{14}$

$$
f_{L}(r)=-\frac{e^{2}}{A\left(4 m_{e} \omega_{L}^{2}\right)} \nabla E_{L}(r)^{2}\left[1-B\left(\cos 2 \omega_{L} t\right)\right] .
$$

For a linearly polarised pulse, $A=B=1$, whereas in the case of circular polarization, $A=2, B=0$, and $t$ is the time. According to Eq. (1), the laser ponderomotive force $f_{L}$ couples the incident photon flux into two primary kinetic modes: (i) hole boring of ions accelerated by the space-charge force associated with electrons under excursion caused by the time-averaged field energy gradients $f_{L} \propto \nabla E_{L}^{2}$ and (ii) relativistic hot electrons excited by the oscillatory component of $f_{L}$ at a frequency of $2 \omega_{L}$.

Formation of collisionless shocks may happen when the piston velocity exceeds the ion sound speed $c_{S}$ $=\sqrt{Z k_{B} T_{e} / m_{i}}$, where $m_{i}$ is the ion mass and $\mathrm{Z}$ is the charge state. The shock velocity can be estimated by comparing the momentum flux of the incoming mass flow with the laserlight pressure. The normalised shock velocity can be written as ${ }^{14}$

$$
v_{\text {shock }} / c=\frac{a_{L}}{\sqrt{2}}\left(\frac{n_{c}}{n_{i}}\right)^{1 / 2}\left(\frac{m_{e}}{m_{i}}\right)^{1 / 2},
$$

where $n_{i}$ is the ion density. The condition for shock formation in terms of the laser-plasma parameter can be expressed as $a_{L}>2 \alpha\left(n_{e} / n_{c}\right),{ }^{14}$ where $\alpha=0.3-0.5$ is the typical 
fraction of laser energy converted to ponderomotive heating of electrons. For our case of $a_{L}=68$ and $n_{e}=40 n_{c}$, this condition is clearly met. On the other hand, according to Eq. (1), the intense laser field pushes the front of the target and generates hot electrons which prevent the Mach number $M=v_{\text {shock }} / c_{s}$ from exceeding the critical value of about 6.5 above which RPA dominates without the formation of a shock. For the laser-plasma parameters considered in this study, $M \simeq 2$ which excludes the possibility of the RPA dominated interaction.

\section{RESULTS}

\section{A. Long driver pulses}

In order to verify the validity and predictive power of our model, first we performed a simulation study with long driver pulses with similar laser-plasma parameters to those of the experiment of Gauthier et al. ${ }^{24}$ The aim of this experiment was the realisation of a proton source with potential for enhanced emittance, flux, and energy. The linearly polarised laser pulse contained 30-50 J energy, the wavelength was $527 \mathrm{~nm}$, and the pulse duration was $\sim 700$ fs (intensity FWHM). After wavefront optimization with a deformable mirror, the pulses were focused by an $f / 3$ off-axis parabola to a spot of 10 to $13 \mu \mathrm{m}$ FWHM. The estimated peak intensity on the target was 3 to $5 \times 10^{19} \mathrm{~W} / \mathrm{cm}^{2}$. The CHM target was produced by a liquid hydrogen jet, cooled down to a temperature of $\sim 17 \mathrm{~K}$, which then frozen with minimal mass loss by evaporation. The result was a continuous $10 \mu \mathrm{m}$ diameter cylinder of solid density $\left(0.08 \mathrm{~g} / \mathrm{cm}^{3}\right)$ hydrogen.

To investigate the ion acceleration mechanism in the cryogenic hydrogen target in view of the experiment of Gauthier et al., ${ }^{24}$ 2D PIC simulations were performed with the PIConGPU code ${ }^{29}$ by using laser and target parameters from the experiment (see above). We show the schematic of the simulation set-up in Figure 1(a) which is similar to that of the experiment. ${ }^{24}$ In the simulation model, 5000 cells along the $\mathrm{y}$-axis and 2048 cells transversely along the $\mathrm{x}$-axis constituted the simulation box.

Figure 2(a) shows the calculated energy distribution of accelerated protons at $2.9 \mathrm{ps}$ after the incidence of laser on the front side of the target. We observe a peak at $\simeq 1.8 \mathrm{MeV}$ in the proton energy spectrum. The density of the peaked proton beam is $0.1 n_{c}$ which is obtained for protons in the $1.5-2.0 \mathrm{MeV}$ energy range. We considered protons in the energy spectrum [shown in Fig. 2(a)], which propagate close to the laser axis (diverging at an angle of $\leq 10^{\circ}$ ). Here, the particle number $d N / d E$ is about $10^{10} / \mathrm{MeV}$, which is close to the proton numbers obtained in the experiment, by the enhanced TNSA scheme for monoenergetic ion beam production. $^{24}$ The broadening of the energy spectrum can be seen due to the TNSA mechanism at the rear side of the target. A spatial density profile of the proton beam is shown in Fig. 2(b). These simulation results, especially the presence of a quasi-monoenergetic peak in the spectrum, support the reasonably good predictive power of our model.

\section{B. Few cycle driver pulses}

To investigate the mechanism of proton acceleration and the involved laser-plasma dynamics with a few-cycle ultraintense laser pulse, we carried out 3D PIC simulations of the interaction of a 20-fs, 2-PW laser pulse with a CHM target. The assumed laser parameters correspond to the PW laser facility at ELI-ALPS which is currently in the development phase. ${ }^{25}$ The scheme of the laser interaction with the CHM target is shown in Fig. 1, as before.

A summary of simulation results is shown in Fig. 3 where the evolution of the longitudinal electric field $[(a)-(c)]$, the electric field of the laser [(d)-(f)], and the ion phase space distribution $[(\mathrm{g})-(\mathrm{i})]$ is illustrated at different time instants of $\mathrm{t}=50 \mathrm{fs}, 87 \mathrm{fs}$, and $100 \mathrm{fs}$. Time instant $t=0$ corresponds to the arrival ( $1 / \mathrm{e}^{2}$ of peak intensity) of the laser pulse at the front surface of CHM. Initially, the laser pulse drives the front surface due to radiation pressure. The critical density surface pushed forward at the hole boring velocity, causing the buildup of an ion density spike (see below). Initially, electrons (followed by protons) are pressed inwards by the radial ponderomotive force of the laser along the laser propagation direction. Thus, the plasma compression due to the radiation pressure provides the required conditions for the shock wave field. As the laser is stopped around the critical density and steepens the plasma profile,
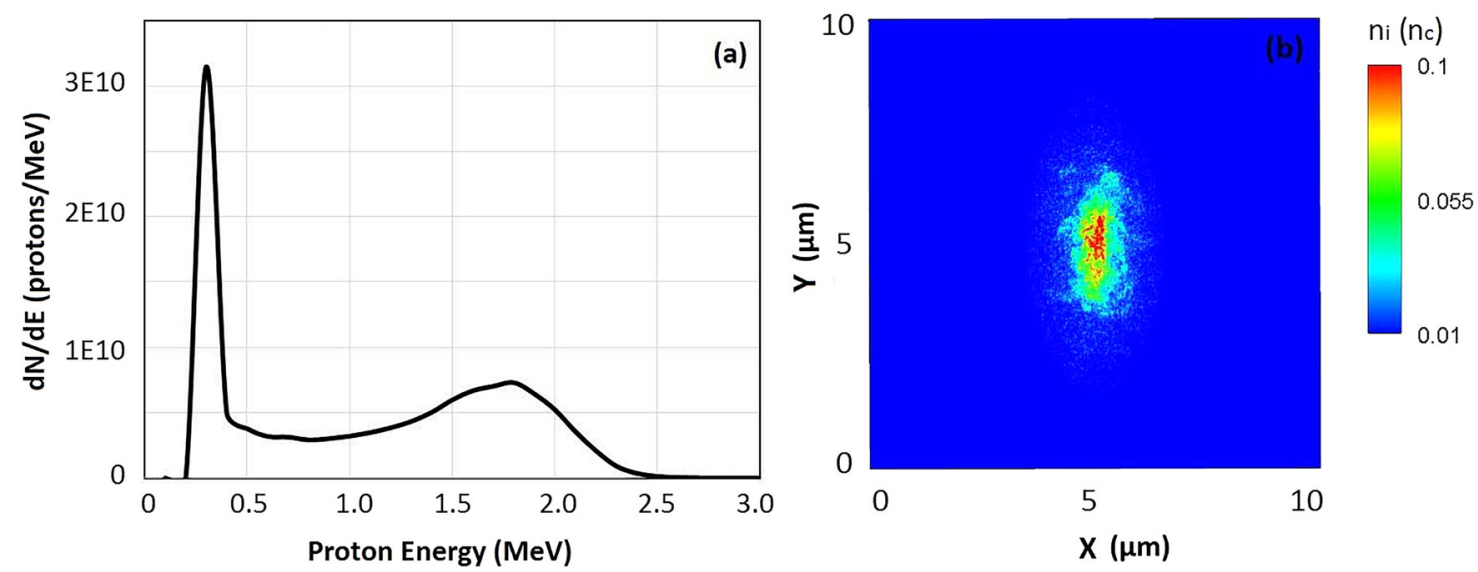

FIG. 2. Simulation results for a long driver pulse interacting with a CHM plasma target. (a) The proton energy distribution for 700 fs driver pulses at 2.9 ps; (b) the spatial distribution of proton energy density with $\leq 10^{\circ}$ of angular divergence. The ion density is normalised with the critical density. 


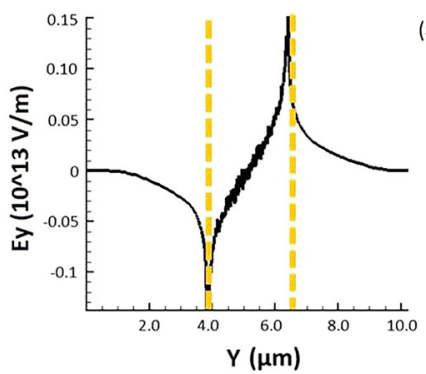

(a)

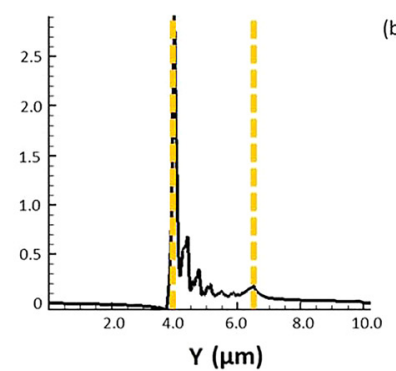

(d)
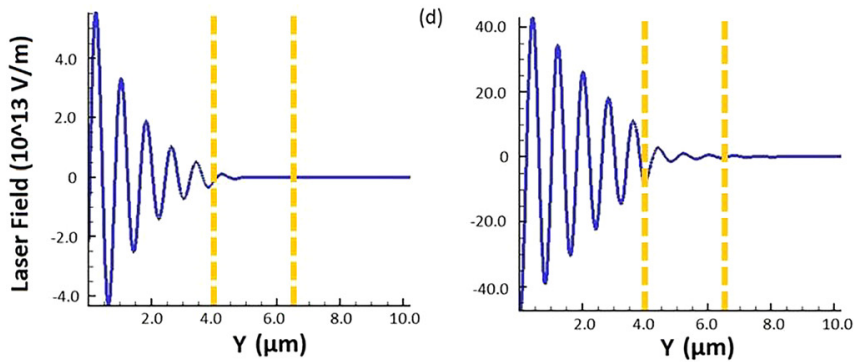

(g)

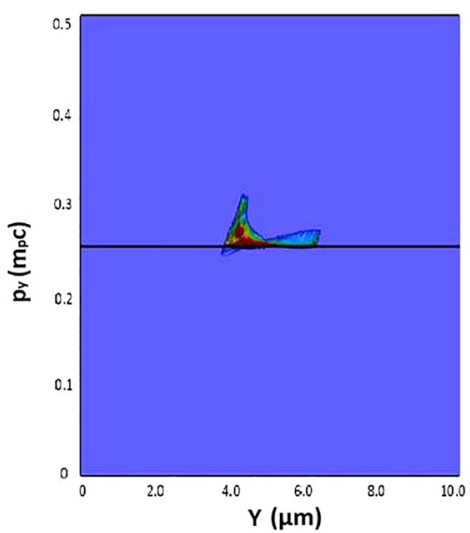

(b)

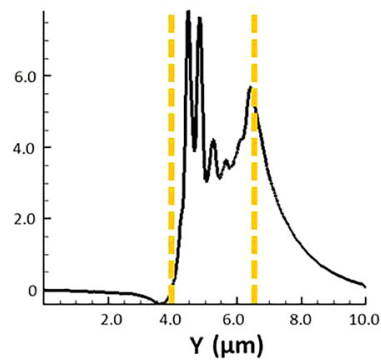

(e)

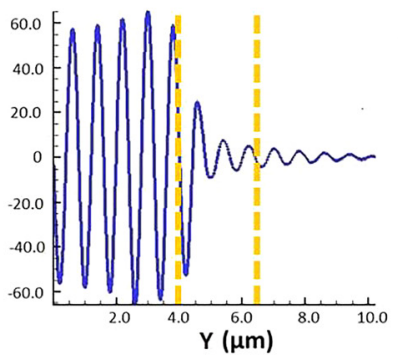

(h)

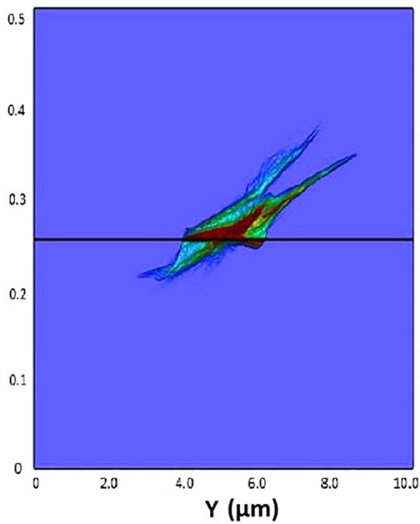

(c)

(f)

(i)

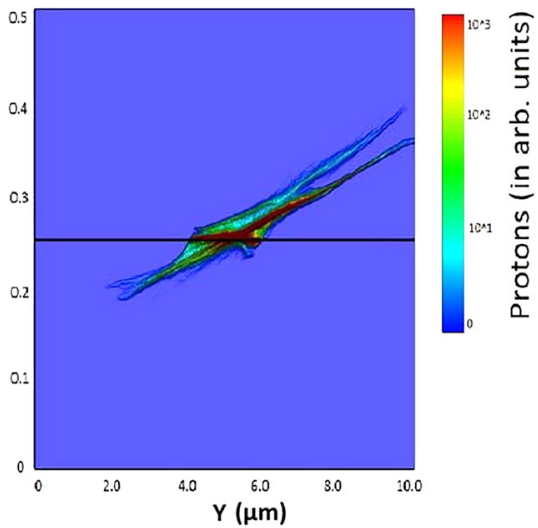

FIG. 3. Simulation results for the few cycle-driver pulse interacting with the CHM plasma target. Evolution of longitudinal electric field (a)-(c), laser field (d)-(f), and ion phase space distribution (g)-(i) for the cylindrical target (length $l_{t}=9 \mu \mathrm{m}$ and diameter $d_{t}=2.5 \mu \mathrm{m}$ ) driven by 20 fs pulses at time instant $t=50 \mathrm{fs}(\mathrm{a}),(\mathrm{d})$, and (g), $87 \mathrm{fs}(\mathrm{b}),(\mathrm{e})$, and (h), and $100 \mathrm{fs}$ (c), (f), and (i). The colour bar shows the ion number density.

the heated electrons propagate through the back side of the target, where they find unperturbed plasma at a similar density, driving a return current that pulls the background electrons to the laser region where they are accelerated. Therefore, thin targets with peak density around the critical density allow for an efficient heating of the entire plasma. The initial build-up of the return current together with the quick recirculation of the heated electrons due to the space charge fields at the front and at the back of the target will lead to a uniform temperature profile, which is crucial in order to have a uniform shock velocity and a uniform ion reflection. The temperature of the electron is consistent with the ponderomotive scaling for the hot electrons, $k_{B} T_{\text {hot }}$ $=25.0 \mathrm{MeV}$. Here, in the case of the intense laser interaction with the target when $n_{e} / \gamma n_{c} \mathrm{w} 0.5$, the strong shock wave field accelerates the ions originating from the front side of the target.

Figure 4 illustrates the evolution of ion density (a) and (c) and magnetic field distribution (b) and (d) at time instant before (a) and (b) and after (c) and (d) shock formation. A strong compression in plasma density is observed between the laser-plasma interface and the shock front and strong filamentation in the upstream region [Figs. 4(a) and 4(b)]. We observe a sharp density modulation of protons near to the front surface of the target. Such a strong density modulation is due to the self-generated electromagnetic field in the plasma. The magnetic field $\left(B_{x}\right)$ distribution in the YZ plane also shows the filamentary structure [Fig. 4(b)], which seems to be generated at the front surface of the target. Filamentation in plasma density and magnetic field can be understood on the basis of counterstreaming currents as mentioned before. In order to observe the propagation of laser accelerated relativistic electrons within the target, local charge neutrality must be maintained. It can be achieved when a much larger return current involving a much larger number of slowly moving electrons is driven by beam to neutralise the fast current density such that $j_{f}+j_{r}=0$, where $j_{f}$ is the fast electron current density and $j_{r}$ is the return current density. The two counterstreaming currents are susceptible for the Weibel unstability. ${ }^{30}$ Since the laser generated electrons are too hot to filament in the background plasma, it is the cold return current which filaments.

Figure 4(c) shows the shock field driven proton density distribution driven by the shock field at $t=87 \mathrm{fs}$, when the 

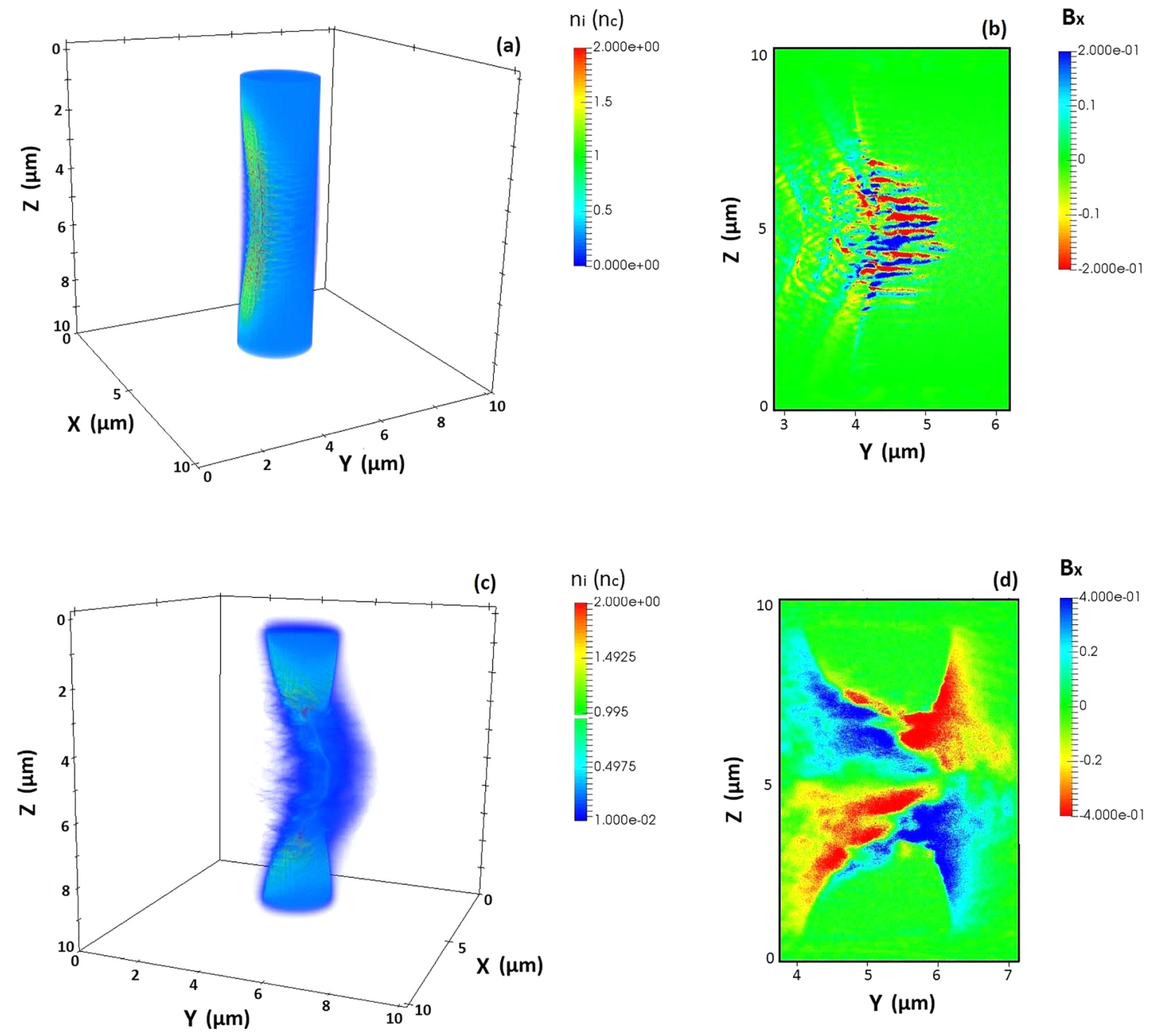

FIG. 4. Evolution of the ion density (a) and (c) and magnetic field distribution (b) and (d) for a few-cycle driver pulse interacting with a CHM plasma target. (a) and (b) refer to the when the peak laser field interacts with the front surface of the target $(t=50 \mathrm{fs}$ ), and (c) and (d) refer to $t=87 \mathrm{fs}$. The colour bars show the variation in ion density (a) and (c) and magnetic field distribution (b) and (d). The ion density $\left(n_{i}\right)$ is expressed in units of critical plasma density $\left(n_{c}\right)$, and the magnetic field $\left(B_{x}\right)$ is in units of Gauss.

shock has been formed and propagates in background plasma. A very strong quasistatic magnetic field of $0.1 \mathrm{G}$ is observed [Fig. 4(d)]. The simulation results agree well with the theoretical estimation of $B=(1 / 2 \pi) \sqrt{\left(\gamma_{r} n_{c} / n_{r}\right)}\left(m_{e} c \omega_{L} / e\right)$ for the magnetic field [26]. Here, $n_{r}$ is the density of return current electrons and $\gamma_{r}$ is the relativistic Lorentz factor of return current electrons, which can be obtained by calculating the Larmor radius of the electrons to the filament wavelength. ${ }^{31}$

In order to understand the evolution of self-generated electric and magnetic fields, we numerically observe the energy associated with these fields as well as the energy absorbed by the plasma particles. Figure 5 shows the temporal evolution of the energy associated with the quasistatic longitudinal electric field $E_{y}$ [Fig. 5(a)], the quasistatic magnetic field $B_{x}$ [Fig. 5(b)], and the plasma particles (electrons and protons) [Fig. 5(c)]. As it can be seen from Fig. 5(a), the energy associated with the electric field is the largest around $50 \mathrm{fs}$. This electric field is associated with the shock wave and reflects a large fraction of the ion population. As the reflected protons propagate further in the plasma while being counterstreamed with the background plasma, a strong magnetic field builds up, as shown in Fig. 5(b). This self-generated quasi-static magnetic field is an important finding in this study, because it deflects the proton beam towards the laser propagation axis and spatially modifies the proton beam distribution. Figure 5(c) depicts the temporal evolution of kinetic energy of electrons and protons.

Figure 6(a) shows the spatial distribution of the proton energy density of the proton beam at time of peak acceleration $(t=100 \mathrm{fs})$. The spatial profile of the proton beam is close to a Gaussian distribution. The beam is collimated due to the magnetic field $\left(B_{x}\right)$ along the laser propagation direction. Figure 6(b) shows the energy spectrum of protons which propagate close to the $y$-axis within a half divergence angle of $10^{\circ}$. The maximum (cut-off) energy obtained in this case is about $160 \mathrm{MeV}$. We also observed a peak at $80 \mathrm{MeV}$ in proton energy distribution which is a characteristic of collisionless shock wave acceleration of protons. The maximum proton energy mainly depends on ions reflected from the shock field, and it can be estimated using Eq. (2) for the shock velocity as ${ }^{16} E_{\text {Proton }}(\mathrm{MeV}) \simeq 74 I\left(10^{21} \mathrm{~W} / \mathrm{cm}^{2}\right) / n_{e}\left(10^{22} \mathrm{~cm}^{-3}\right)$. The maximum proton energy obtained from this formula is about $125 \mathrm{MeV}$, which is less than the simulation results of $160 \mathrm{MeV}$. The enhancement in maximum proton energy 
(a)

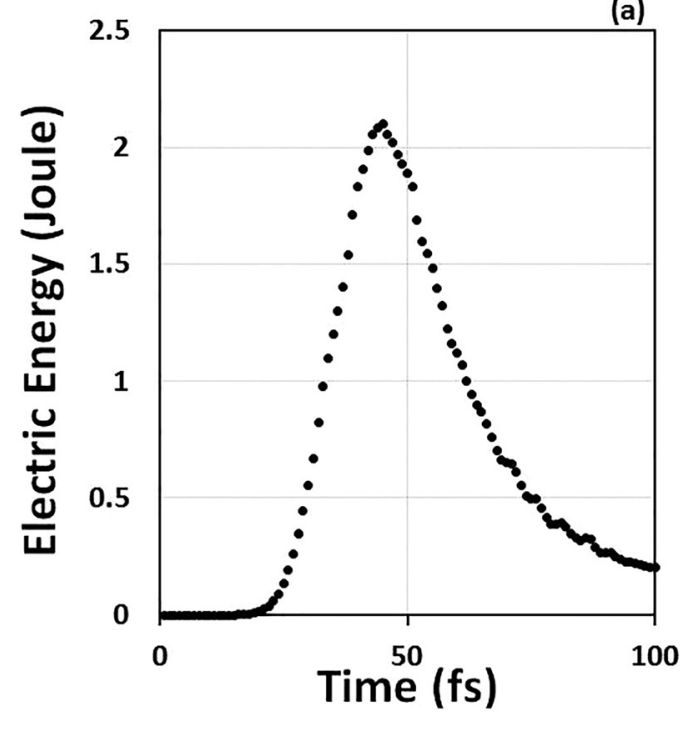

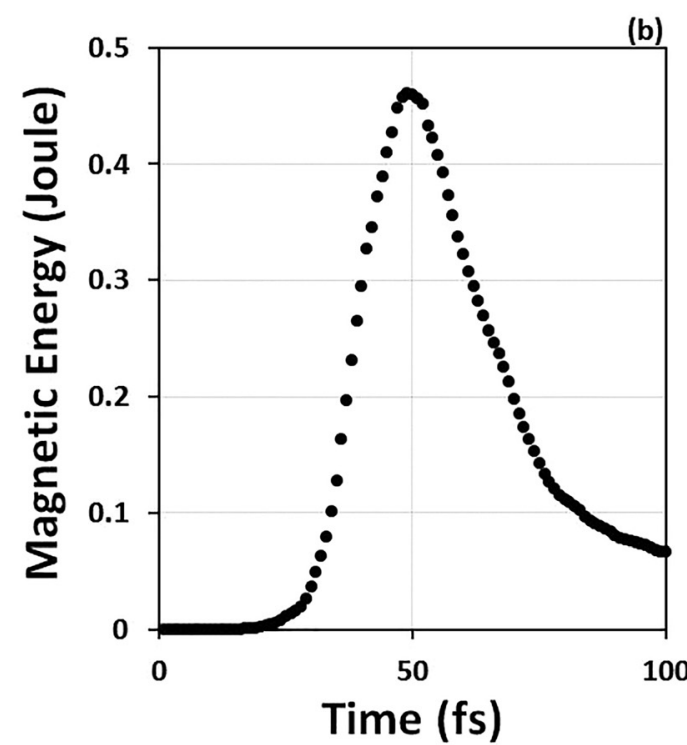

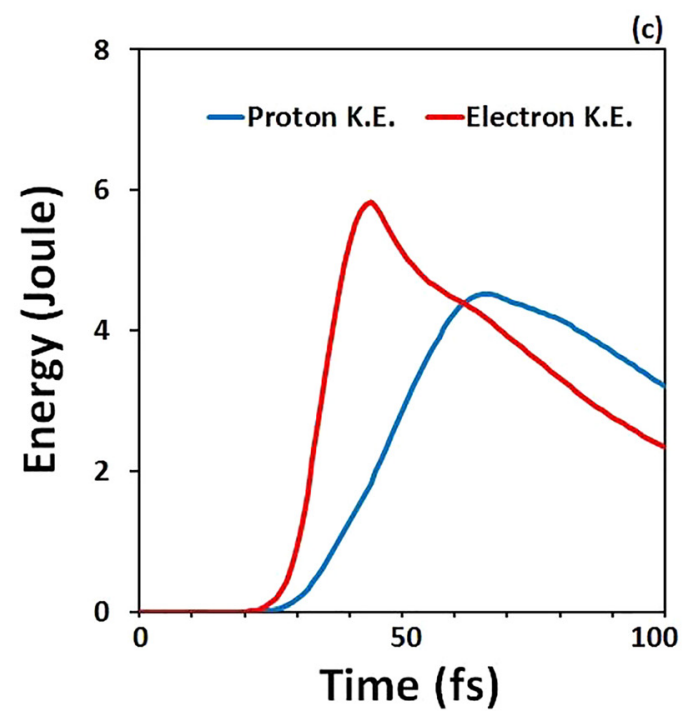

FIG. 5. Temporal evolution of the energy of the quasistatic electric field $\left(E_{y}\right)(a)$, magnetic field $\left(B_{x}\right)(\mathrm{b})$, and of the kinetic energy associated with the electrons and protons.

observed in the simulation can be seen as an additional acceleration at the rear surface of the target due to quasi-static electric and magnetic field [as shown in Figs. 3(c) and 4(d)]. It is interesting to note that the analytical formula given above predicts a maximum proton energy up to $250 \mathrm{MeV}$ for a focused laser intensity of $2 \times 10^{22} \mathrm{~W} / \mathrm{cm}^{2}$ on the target, which is very promising for medical applications.

The spatial distribution of proton energy density shows that high-energy protons are collimated along the laser propagation direction within a small area. Besides peak proton energy, another important point in terms of applications is the conversion efficiency of laser energy to the proton beam. This can be estimated from the ratio of the total proton energy to the laser energy. For the target size of $d_{t}=2.5 \mu \mathrm{m}$, we observed a conversion efficiency of $\simeq 1 \%$ for $10^{10}$ protons with energy higher than $10 \mathrm{MeV}$, accelerated along the laser propagation direction.

Figure 7 shows the temporal evolution of laser pulse energy (dashed lines) and the kinetic energy of accelerated protons (solid lines). The 3D simulations performed for different target diameters of $1 \mu \mathrm{m}, 2.5 \mu \mathrm{m}$, and $5 \mu \mathrm{m}$ (with the same laser-plasma parameters as before) clearly show a sensitive dependence of the total proton energy on the target size (Fig. 7). Therefore, the optimal choice of the laser beam size and target diameter is critical for transporting maximum energy from the laser field to the protons. We found that a laser beam size slightly larger than the target diameter resulted in a higher proton energy. For our 5- $\mu \mathrm{m}$ diameter laser beam, the highest efficiency was achieved with the $2.5-\mu \mathrm{m}$ diameter target.

It shall be noted that the presence of a preplasma can influence the laser-plasma interaction dynamics. 2D3V PIC simulations were performed to estimate the preplasma effects on proton acceleration. We numerically varied the preplasma scale length between 0 and $10 \mu \mathrm{m}$ to investigate its relevance for proton energy distributions. The results show that for a preplasma of about the critical scale length $0.13 \lambda_{L} a_{0}^{0.5}=0.88 \mu \mathrm{m}$ or shorter, there is only a small and smooth variation of the proton density at the rear surface. 

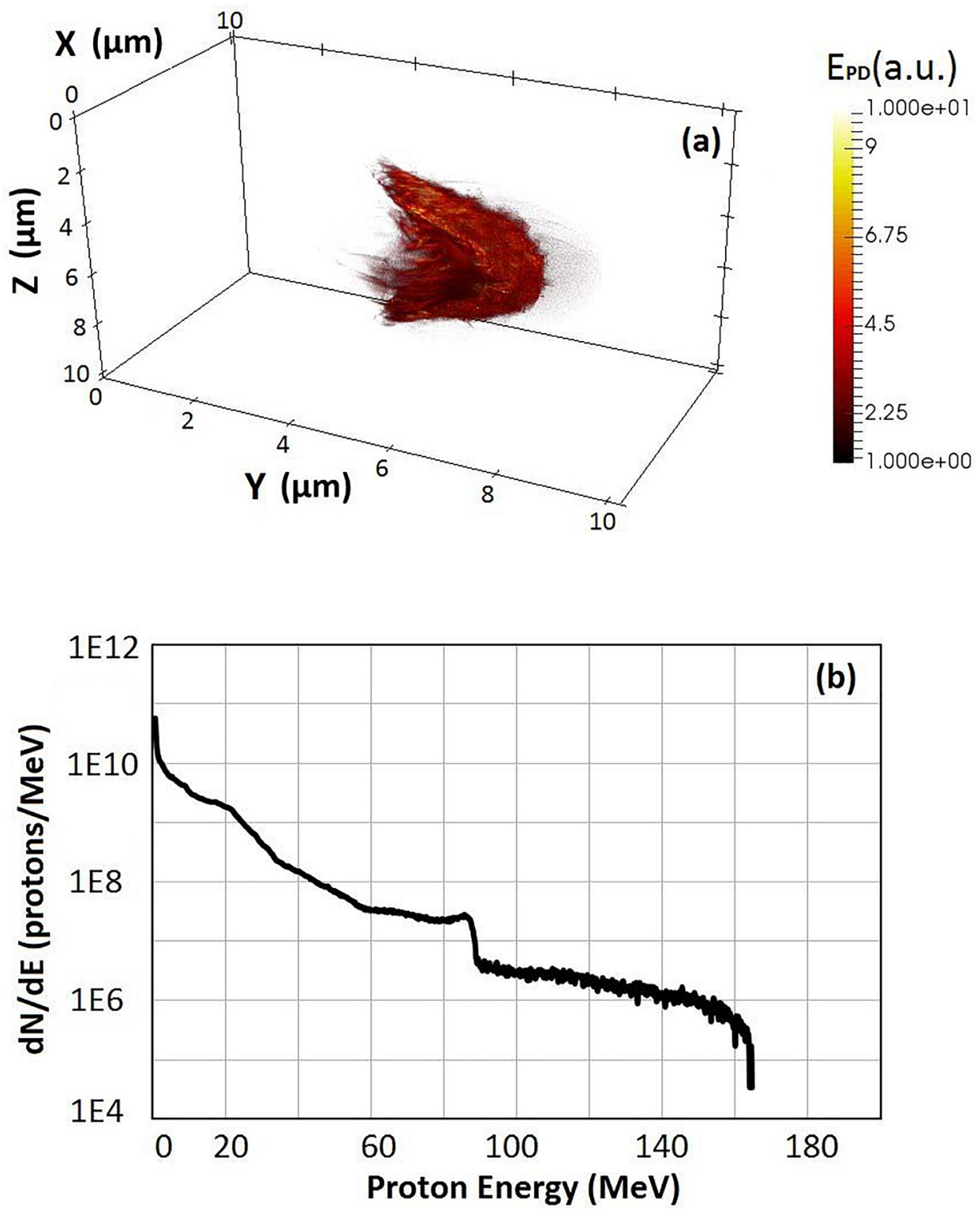

FIG. 6. Spatial distribution of proton energy density and proton energy distribution. (a) Spatial distribution of proton energy density for target diameter $d_{t}=2.5 \mu \mathrm{m}$; (b) energy spectrum at $t=100 \mathrm{fs}$ of protons accelerated along the laser propagation direction within a divergence angle of $10^{\circ}$.
These findings are in agreement with the recent experimental and simulation studies. ${ }^{26}$ The dependence of the maximum (cut-off) proton energy on the preplasma length is shown in Fig. 8. We find that a preplasma up to about the critical length does not show a significant effect on the peak proton energy. However, a preplasma over the critical length can significantly reduce the proton energy.

Recent experimental results ${ }^{28}$ with the CHM target have achieved a maximum proton energy of $20 \mathrm{MeV}$ with $2.6 \mathrm{~J}$ of laser energy, where it has also been observed that the proton energy was limited by the preplasma effect. This is in good agreement with our simulation results (as shown by Fig. 8). Thus, based on the most recent experiment results ${ }^{27,28}$ and the results illustrated in this study, we can expect that future experiments with higher pulse energies and precisely controlled prepulses can deliver higher proton energies.

\section{CONCLUSION}

Laser-driven proton acceleration from mass-limited cryogenic hydrogen microjet targets was numerically investigated for long (700 fs) and few-cycle (20 fs) laser pulses. For long pulses, we numerically observed a quasimonoenergetic proton peak at $1.8 \mathrm{MeV}$, in reasonable accordance with the experimental finding of Gauthier et $a .^{24}$ In order to achieve higher proton energy, a new regime of proton acceleration was numerically investigated by using 20 -fs pulses with a 2-PW peak power and $10^{22} \mathrm{~W} / \mathrm{cm}^{2}$ focused intensity with controlled prepulses. Such a high-power fewcycle laser will be available in the near future, for example, at ELI-ALPS. ${ }^{25}$ Proton energies as high as $160 \mathrm{MeV}$ were predicted by simulations with a quasi-monoenergetic peak around $80 \mathrm{MeV}$. This peak is characteristic for collisionless shock-wave acceleration. It is observed that the proton energy can be maximised when the laser beam size becomes slightly larger than the target diameter. A very strong quasistatic magnetic field of a few Gauss was observed, which modulates the spatial density profile of the proton beam along the laser propagation direction. Such strong quasistatic magnetic fields may allow us to study the astrophysical process at advanced laser facilities. A preplasma of length over the critical value ${ }^{26}$ can reduce the proton energy, but 


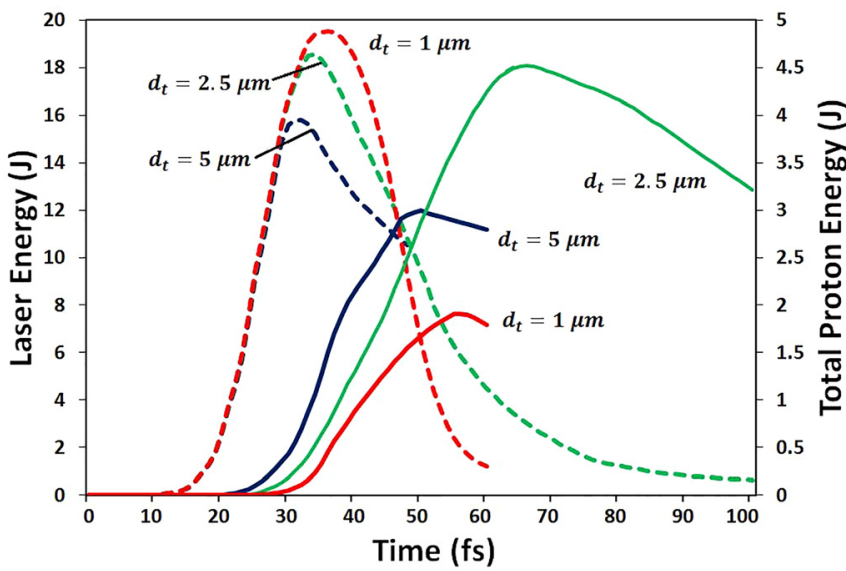

FIG. 7. Temporal evolution of laser pulse energy (dashed lines) and kinetic energy of protons (solid lines) for different target diameters of $d_{t}=1 \mu \mathrm{m}$ (red), $2.5 \mu \mathrm{m}$ (green), and $5.0 \mu \mathrm{m}$ (blue). The laser beam parameters were the same as in the previous simulations.

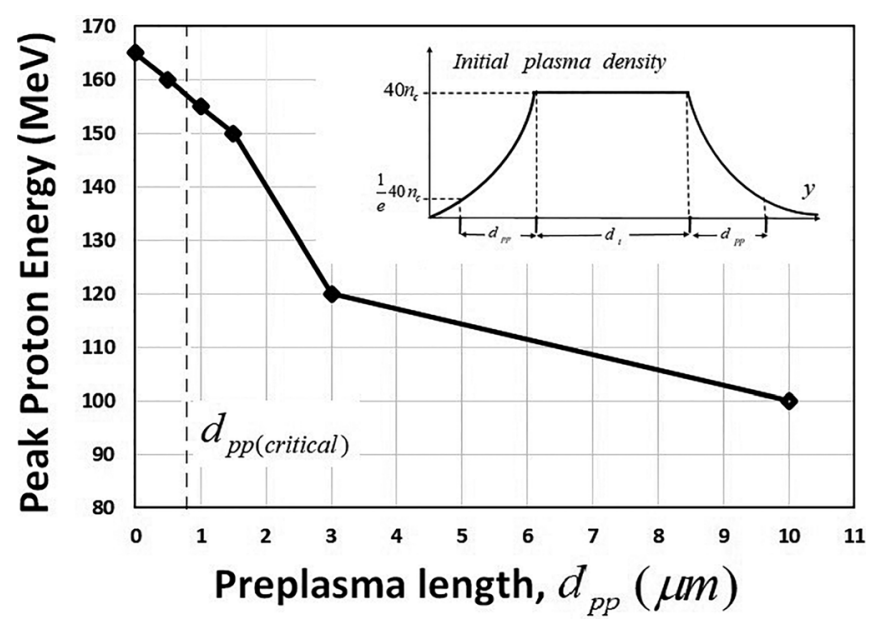

FIG. 8. Dependence of peak proton energy on the preplasma scale length. Laser-plasma parameters are the same as in previous simulation results.

the use of a double plasma mirror can mitigate such effects. Even higher maximum proton energies up to $250 \mathrm{MeV}$ and beyond can be expected on the basis of simulation results in this work and analytical estimations ${ }^{16}$ by focusing the laser to still higher intensities of $2 \times 10^{22} \mathrm{~W} / \mathrm{cm}^{2}$ on the target. Such high proton energies are required for cancer therapy to treat deep-seated tumors. ${ }^{1}$

\section{ACKNOWLEDGMENTS}

We performed PIC simulations utilizing the open-source code PIConGPU version $0.1 .2^{29}$ in this research. We acknowledge support of the Department of Information Services and Computing, Helmholtz-Zentrum DresdenRossendorf (HZDR), Germany, for providing access to the GPU Compute Cluster Hypnos. This work was funded as part of the European Cluster of Advanced Laser Light Sources (EUCALL) project which has received funding from the European Union's Horizon 2020 research and innovation programme under Grant Agreement No. 654220. This work was supported in part by the Hungarian Scientific Research Fund (OTKA) under Grant No. 113083. J. A. Fülöp acknowledges support from a Janos Bolyai Research Scholarship (Hungarian Academy of Sciences). The present scientific contribution is dedicated to the 650th anniversary of the foundation of the University of Pécs, Hungary.

${ }^{1}$ V. Malka, J. Faure, Y. A. Gauduel, E. Lefebvre, A. Rousse, and K. Ta. Phuoc, Nat. Phys. 4, 447 (2008).

${ }^{2}$ E. L. Clark, K. Krushelnick, J. R. Davies, M. Zepf, M. Tatarakis, F. N. Beg, A. Machacek, P. A. Norreys, M. I. K. Santala, I. Watts, and A. E. Dangor, Phys. Rev. Lett. 84, 670 (2000).

${ }^{3}$ A. Maksimchuk, S. Gu, K. Flippo, D. Umstadter, and V. Yu. Bychenkov, Phys. Rev. Lett. 84, 4108 (2000).

${ }^{4}$ R. A. Snavely, M. H. Key, S. P. Hatchett, T. E. Cowan, M. Roth, T. W. Phillips, M. A. Stoyer, E. A. Henry, T. C. Sangster, M. S. Singh, S. C. Wilks, A. MacKinnon, A. Offenberger, D. M. Pennington, K. Yasuike, A. B. Langdon, B. F. Lasinski, J. Johnson, M. D. Perry, and E. M. Campbell, Phys. Rev. Lett. 85, 2945 (2000).

${ }^{5}$ S. C. Wilks, A. B. Langdon, T. E. Cowan, M. Roth, M. Singh, S. Hatchett, M. H. Key, D. Pennington, A. MacKinnon, and R. A. Snavely, Phys. Plasmas 8, 542 (2001).

${ }^{6}$ P. Mora, Phys. Rev. Lett. 90, 185002 (2003).

${ }^{7}$ J. Fuchs, P. Antici, E. d'Humieres, E. Lefebvre, M. Borghesi, E. Brambrink, C. A. Cecchetti, M. Kaluza, V. Malka, M. Manclossi, S. Meyroneinc, P. Mora, J. Schreiber, T. Toncian, H. Pepin, and P. Audebert, Nat. Phys. 2, 48 (2006).

${ }^{8}$ L. Robson, P. T. Simpson, R. J. Clarke, K. W. D. Ledingham, F. Lindau, O. Lundh, T. McCanny, P. Mora, D. Neely, C.-G. Wahlström, M. Zepf, and P. McKenna, Nat. Phys. 3, 58 (2007).

${ }^{9}$ T. E. Cowan, J. Fuchs, H. Ruhl, A. Kemp, P. Audebert, M. Roth, R. Stephens, I. Barton, A. Blazevic, E. Brambrink, J. Cobble, J. Fernández, J.-C. Gauthier, M. Geissel, M. Hegelich, J. Kaae, S. Karsch, G. P. L. Sage, S. Letzring, M. Manclossi, S. Meyroneinc, A. Newkirk, H. Pépin, and N. Renard-LeGalloudec, Phys. Rev. Lett. 92, 204801 (2004).

${ }^{10}$ S. C. Wilks, W. L. Kruer, M. Tabak, and A. B. Langdon, Phys. Rev. Lett. 69, 1383 (1992).

${ }^{11}$ A. P. L. Robinson, P. Gibbon, M. Zepf, S. Kar, R. G. Evans, and C. Bellei, Plasma Phys. Controlled Fusion 51, 024004 (2009).

${ }^{12}$ C. A. J. Palmer, N. P. Dover, I. Pogorelsky, M. Babzien, G. I. Dudnikova, M. Ispiriyan, M. N. Polyanskiy, J. Schreiber, P. Shkolnikov, V. Yakimenko, and Z. Najmudin, Phys. Rev. Lett. 106, 014801 (2011).

${ }^{13}$ T. Esirkepov, M. Borghesi, S. V. Bulanov, G. Mourou, and T. Tajima, Phys. Rev. Lett. 92, 175003 (2004).

${ }^{14}$ A. Macchi, F. Cattani, T. V. Liseykina, and F. Cornolti, Phys. Rev. Lett. 94, 165003 (2005).

${ }^{15}$ L. O. Silva, M. Marti, J. R. Davies, R. A. Fonseca, C. Ren, F. S. Tsung, and W. B. Mori, Phys. Rev. Lett. 92, 015002 (2004).

${ }^{16}$ D. Haberberger, S. Tochitsky, F. Fiuza, C. Gong, R. A. Fonseca, L. O. Silva, W. B. Mori, and C. Joshi, Nat. Phys. 8, 95 (2012).

${ }^{17}$ F. Fiuza, R. A. Fonseca, J. Tonge, W. B. Mori, and L. O. Silva, Phys. Rev. Lett. 108, 235004 (2012).

${ }^{18}$ F. Fiuza, A. Stockem, E. Boella, R. A. Fonseca, L. O. Silva, D. Haberberger, S. Tochitsky, W. B. Mori, and C. Joshi, Phys. Plasmas 20, 056304 (2013)

${ }^{19}$ A. Henig, D. Kiefer, K. Markey, D. C. Gautier, K. A. Flippo, S. Letzring, R. P. Johnson, T. Shimada, L. Yin, B. J. Albright, K. J. Bowers, J. C. Fernández, S. G. Rykovanov, H.-C. Wu, M. Zepf, D. Jung, V. K. Liechtenstein, J. Schreiber, D. Habs, and B. M. Hegelich, Phys. Rev. Lett. 103, 045002 (2009).

${ }^{20}$ D. Jung, L. Yin, B. J. Albright, D. C. Gautier, S. Letzring, B. Dromey, M. Yeung, R. Hörlein, R. Shah, S. Palaniyappan, K. Allinger, J. Schreiber, K. J. Bowers, H. C. Wu, J. C. Fernandez, D. Habs, and B. M. Hegelich, New J. Phys. 15, 023007 (2013).

${ }^{21}$ L. Yin, B. J. Albright, K. J. Bowers, D. Jung, J. C. Fernandez, and B. M. Hegelich, Phys. Rev. Lett. 107, 045003 (2011).

${ }^{22}$ M. Roth, D. Jung, K. Falk, N. Guler, O. Deppert, M. Devlin, A. Favalli, J. Fernandez, D. Gautier, M. Geissel, R. Haight, C. E. Hamilton, B. M. Hegelich, R. P. Johnson, F. Merrill, G. Schaumann, K. Schoenberg, M. Schollmeier, T. Shimada, T. Taddeucci, J. L. Tybo, F. Wagner, S. A. Wender, C. H. Wilde, and G. A. Wurden, Phys. Rev. Lett. 110, 044802 (2013).

${ }^{23}$ A. P. L. Robinson, M. Zepf, S. Kar, R. G. Evans, and C. Bellei, New J. Phys. 10, 013021 (2008). 
${ }^{24}$ M. Gauthier, J. B. Kim, C. B. Curry, B. Aurand, E. J. Gamboa, S. Göde, C. Goyon, A. Hazi, S. Kerr, A. Pak, A. Propp, B. Ramakrishna, J. Ruby, O. Willi, G. J. Williams, C. Rödel, and S. H. Glenzer, Rev. Sc. Instr. 87, 11D827 (2016).

${ }^{25}$ See http://www.eli-alps.hu for ELI-ALPS.

${ }^{26} \mathrm{~S}$. Gode, C. Rödel, K. Zeil, R. Mishra, M. Gauthier, F.-E. Brack, T. Kluge, M. J. MacDonald, J. Metzkes, L. Obst, M. Rehwald, C. Ruyer, H.-P. Schlenvoigt, W. Schumaker, P. Sommer, T. E. Cowan, U. Schramm, S. Glenzer, and F. Fiuza, Phys. Rev. Lett. 118, 194801 (2017).

${ }^{27}$ M. Gauthier, C. B. Curry, S. Gode, F. E. Brack, J. B. Kim, M. J. MacDonald, J. Metzkes, L. Obst, M. Rehwald, C. Rodel, H. P. Schlenvoigt, W. Schumaker, U. Schramm, K. Zeil, and S. H. Glenzer, Appl. Phys. Lett. 111, 114102 (2017).
${ }^{28}$ L. Obst, S. Gode, M. Rehwald, F. E. Brack, J. Branco, S. Bock, M. Bussmann, T. E. Cowan, C. B. Curry, F. Fiuza, M. Gauthier, R. Gebhardt, U. Helbig, A. Huebl, U. Hubner, A. Irman, L. Kazak, J. B. Kim, T. Kluge, S. Kraft, M. Loeser, J. Metzkes, R. Mishra, C. Rodel, H. P. Schlenvoigt, M. Siebold, J. Tiggesbaumker, S. Wolter, T. Ziegler, U. Schramm, S. H. Glenzer, and K. Zeil, Sci. Rep. 7, 10248 (2017).

${ }^{29}$ M. Bussmann, H. Burau, T. E. Cowan, A. Debus, A. Huebl, G. Juckeland, T. Kluge, W. E. Nagel, R. Pausch, F. Schmitt, U. Schramm, J. Schuchart, and R. Widera, in Proceedings of International Conference on High Performance Computing, Networking, and Storage Analysis (2013), Vol. 5, p. 1.

${ }^{30}$ E. S. Weibel, Phys. Rev. Lett. 2, 83 (1959).

${ }^{31}$ R. C. Davidson, D. A. Hammer, I. Haber, and C. E. Wagner, Phys. Fluids 15, 317 (1972) 